\title{
Bridging of different sites by bohemians and tourists: analysis by agent-based simulation
}

\author{
Shiro Horiuchi ${ }^{1}$ iD
}

Received: 18 May 2020 / Accepted: 15 November 2020 / Published online: 2 December 2020

(C) The Author(s) 2020

\begin{abstract}
Individuals often aggregate in areas of high density where they can form profitable social networks. Individuals of few resources cannot manage the high costs of density and are displaced into areas of low density. The lifestyle of bohemian and tourist may increase the profits of all parties and shrink inequalities among sites. Since these lifestyles are possible only for the rich, this phenomenon may further expand inequality. To test the effects of the lifestyle of bohemian and tourist on inequality, we run an agent-based simulation (ABS) in which some agents (individuals) select only their residence sites (singular) and others select two sites for their residence and visits (dual), paying additive costs. The ABS demonstrates that when we increase the number of duals, all agents gain higher profits, and inequalities among agents of different sites decrease. The ABS also demonstrates that any agent evolves to a dual when the costs of density and travel are small. Further research could consider the possibility of the dual lifestyle by conducting studies on groups of bohemians and tourists.
\end{abstract}

Keywords Density $\cdot$ Travel $\cdot$ Social network $\cdot$ Globalization $\cdot$ Inequality

\section{Introduction}

Social networks affect individuals' behaviors; in turn, individuals affect their social network. If a social network evolves thorough the mechanism of self-organization, naïve intuition is unlikely to predict the outcomes of a social network. Computer simulations have contributed to reproducing the dynamics of self-organization. Various disciples, including the social sciences [9], have adopted computer simulations to analyze the evolution of social networks.

Most studies on social networks have focused on preferential attachment for the evolution of social networks. Scale-free networks appear if nodes are linked with

Shiro Horiuchi

horiuchi.shiro@gmail.com

1 Faculty of International Tourism, Hannan University, Matsubara City, Japan 
other nodes, depending on the number of links that the nodes already have. Scalefree networks explain actor collaborations, power grids, and the World Wide Web [4]. Succeeding studies have assumed additive realistic conditions in the network formation rule for individuals' choice; individuals are assumed to seek their links by their homophily [34], transitive ties [49], betweenness rather than degree [60], or the position of structural holes [13]. In these studies, links form globally, with small or no costs across space.

Individuals usually cannot link to others in just any place. In areas where the population density is too low or individuals are too far apart, they probably will not find others to link to. In other words, spatially proximate individuals will probably become members of the same network. The classical study demonstrates that friendships are more likely formed among proximate neighbors [44]; subsequent studies have also demonstrated that friendships are locally formed, even if formed over the internet [35, 38]. An individual could choose favorable fellows with shared interest, profession, ethnicity, sexual orientation, or else, in urban areas where many individuals aggregate [54].

Locally formed social networks often favor individuals. Aggregation of cooperation can expand, excluding defectors [2, 45]. Local aggregation of cooperation represents local communities in the real world. The members monitor and punish free riders [46]. Regarding economic growth, the aggregation of individuals results in increasing returns [36]. Interaction among individuals creates communities and roads that further facilitate the aggregation of individuals, strengthen social ties, and promote innovation in the areas [8, 37, 47]. Firms perform well in research and development or innovation when located nearby other firms [24]. Researchers are more likely to engage in collaboration if their affiliations are proximately located [7]. In inner-city areas, including slums, residents help one another and start new business because of the network among the residents [30]. In developing countries, migration often occurs from rural areas to inner-city areas, and even when jobs are scarce in inner-city areas, the probability of obtaining a job is higher than that in rural areas [39, 59]. Accordingly, if individuals want to form networks with other individuals through which they can profit, the former should escalate into areas of aggregation, which further increases population density and proximate interaction in the areas. Based on these findings, an assumption of studies has been that proximity is the necessary condition for network formation [1, 15, 41].

Not all individuals can reside in areas of high population density. Negative consequences such as long commute times, crime, and infectious diseases accumulate in urban areas because of high population density. Super-rich individuals and large transnational companies aggregate in global cities where industries of information, finance, and professional services prosper [53]. Using the gap between actual and potential estate rate, global capital develops inner-city areas. Neo-liberal or authoritarian governments facilitate the development. Long-time residents, often poor, are displaced from urban areas to suburban or rural areas; this is considered to be the main negative consequence of gentrification [57]. These displaced individuals no longer make money from their rich neighbors, as they did in urban areas, and become further deprived. A classical study shows the mechanism of individuals' migration and segregation based on ethnic groups [55]. Succeeding studies have 
demonstrated segregation among individuals on the basis of wealth $[6,32,51,52$, 61]; rich residents moving away from poor areas, or poor residents' exclusion from rich areas. Hence, inequalities among regions in countries expand as globalization proceeds [20].

Another result of globalization is that an increasing number of individuals can pursue bohemian and tourist lifestyles. The lifestyle may counter-expand the inequality among locations. Florida [21] demonstrated the possibility that the bohemian lifestyle affects the drivers of economic growth. These lifestyles are expected to promote tolerance toward marginalized individuals, including foreigners and outsiders. Tourists also shrink inequalities among locations by cooperating with poor residents of exploited areas [22]. McIntyre et al. [43] reviewed case studies of multiple-site residence in developed countries. Multiple-site residents may increase profits and decrease the inequalities among all individuals by forming a social network that bridges locations. In Japan, depopulation is serious. That is partly because too many individuals live in the capital city Tokyo with the lowest fertility rate of all cities in Japan [42]. The Japanese government facilitates dual-site residence as a program for regional revitalization and to decelerate the swiftest depopulation.

The literature usually has assumed only one site per individual; however, as described, individuals may select multiple sites as a residence or place to visit. Watts and Strogatz [62] demonstrated how local clustering and short global separation are possible using a small-world network that assumes a few random pathways between distant areas. A long path between distant areas may represent links caused by agents with multiple sites. All individuals may benefit from links along the long path across different sites, similar to the benefits of bridging social capital for all members [50]. By contrast, only rich individuals can avail such lifestyles and increase their profits. Bauman [5] asserted that the modern age is characterized by gross inequalities between rich and poor individuals. Rich individuals are freed from local communities but restricted by time. Poor individuals have lost their communities because of geographical mobility and the fluidity of contemporary society.

Thus, whether multiple selection for residences and visits decreases or increases inequality among locations is a complicated problem. We cannot expect an outcome by applying only naïve intuition. In this study, we use an agent-based simulation (ABS) because it is useful to analyze the dynamics of self-organizing social networks.

\section{Model}

The ABS assumed $N$ agents, and each agent has one residence site along a line of $M$ sites. In the present simulation, the number of agents $N$ is 100 . The number of sites $M$ is 10 , with two boundaries; each site is numbered from 1 to 10 ; sites 1 and 10 are the two boundaries. Agents change their residence sites to increase their profits along turns. Some agents also have their visit sites; each of them selects one site to visit to increase her profit; therefore, each has two sites for residence and visits. Hereafter, we call agents with only residence sites singulars and agents with two sites either for residence or visits duals. 
Each agent has a set of resources beyond which she cannot sustain more links, more population density, and more travel, as described in this paragraph. The resource limit is $w_{i}$ for agent $i$. The value is integral within the range of $6 \leq w \leq 15$. We refer to $w$ to categorize agents as poor or rich. The number of agents with each resource $(w=6,7, \ldots 15)$ is 10 ; in total, there are 100 agents. We calculate the number of agents at site $k$ as $n_{k}$, and that satisfies $0 \leq n_{k} \leq 100$ for any $k$, and $\sum n_{k}=100$. Agents at site $k$ suffer the cost of density as $p n_{k}$; the value $p$ represents the cost of density per population. The cost, $p$ times population, implies estate rate, commuting time, risk of infectious diseases, and so forth, which should increase as the population increases.

We assume agents have their upper limit of links. This assumption is in line with Jackson and Wolinsky [31] in the sense that agents pay opportunity costs by forming links with others. Dunbar [19] predicted that social groups of human beings can at most consist of some 100s, which is extrapolated from the regression line between neocortex size and social group sizes of primate species. We also assume individuals do not select companion links directly but rather choose sites where they can link with others. Individuals are linked by chance, based on their residence or visit sites. In the present model, agents do not know in advance how they will form links or with whom. Agents only select the site where their possible linked others reside or visit. Models in the literature have also assumed probabilistic network formation between agents [56]. In this study, complete graphs for agents are formed at each site. Next, we select all agents by random order. For each agent $i$ cut his links with agent $j$, as long as either of the following equations holds.

$$
\begin{aligned}
& v_{i}>w_{i}-p n_{k}, \\
& v_{j}>w_{j}-p n_{k} .
\end{aligned}
$$

Agent $j$ is selected by random order among all the links of agent $i$. The values $v_{i}$ and $v_{j}$ represent the number of links of agent $i$ or $j$, respectively. The right side of the equations represents their resources $\left(w_{i}\right.$ and $w_{j}$ ) minus the cost of density, as aforementioned. The procedures continue until Eqs. (1.1) and (1.2) hold for all agents.

Agents are assumed to gain profits from links, such as various sources of information, support, and community services from linked others. If agents are not linked directly, but indirectly until the fourth degree, they are assumed to gain additive profits. We count the number of agent $i$ 's first, second, third, or fourth neighbors as $l_{1-i}, l_{2-i}, l_{3-i}$, and $l_{4-i}$. Her profit $z_{i}$ is calculated as follows:

$$
z_{i}=l_{1-i}+l_{2-i} f+l_{3-i} f^{2}+l_{4-i} f^{3} .
$$

In Eq. (2), $f$ represents the discounted ratio of indirect links $(0<f<1)$. A higher value of $f$ means higher profits from indirect links. We set the limits of indirect links until the fourth, which may be sufficient to count the profits of indirect links. By decreasing the value of $f$, we can minimize the effects of distant links for profits. We regard the number of links to be proportional to the profits of agents. Although other profits may be possible using the topology of the network [13], the total number of links should be appropriate if we expect not only 
economic gains but also social support from others. In the classical study [4], agents with more links gain further links; thus, we might assume that many links are proportional to the profits of agents.

Initially, all agents are distributed randomly at each of the ten sites. Duals select the same sites for residence and visits. At each turn, one agent is randomly selected to change her site, either that for residence or visits. If the selected agent is singular, she randomly selects one site among sites and migrates there, unless the density cost is too large for her to remain in the area. Agent $i$ loses her original links in the former site and forms links at the migration site $j$ up to her maximum resources minus density costs as follows:

$$
v_{i}<w_{i}-p n_{i}
$$

Agents may link with both singulars and duals, or residence or visits. The agent's profits are calculated as the temporary after migration. Unless the profit increases after migration, the migration is canceled; the selected agent moves back to the original site, and the original links recover. Other agents do not suffer additive costs from this procedure.

If the selected agent $i$ is dual, she randomly selects one site among sites. She visits the selected site unless the density and travel costs are too great. The agent loses all her links at first. Next, she forms links both at her residence site $j$ and the visit site $k$ up to her maximum resources minus density costs at two sites and travel cost as follows:

$$
v_{i}<w_{i}-p n_{j}-p n_{k}-q\left|x_{j}-x_{k}\right| .
$$

She may link with either singulars or duals. Although duals suffer a density cost at their visit site, their visit is not counted as a residence for the other agents ( $n_{k}$ excludes the agent $i$ ). The value $q$ represents the cost of travel per distance. The number of $x_{j}$ and $x_{k}$ represents the location of sites $j$ and $k\left(1 \leq x_{j}, x_{k} \leq 10\right)$; hence, $\left|x_{j}-x_{k}\right|$ represents the distance between the two sites. As the cost (i.e., $q$ times distance) increases, duals must pay more to maintain two sites, one each for residence and visits. If $j$ is identical to $k$, or they are the same site, either $p n_{j}$ or $p n_{k}$, and $q\left|x_{j}-x_{k}\right|$ is counted as zero. The agent's profits are calculated as the temporary after the visit change. Unless the profit increases after the visit, the visit is canceled; the selected agent returns to the former site, and the original links recover. After the procedure, the selected agent exchanges her residence site with her visit site by the probability 0.5 . Therefore, duals change their visit sites and residence sites over time.

At each 100 turns, all links are checked. If any agents hold too many links over their resources, the links are cut unless Eqs. (3) or (4) hold. We calculate the actual profits for all agents, instead of temporary profits. We assume 100 turns to be about 1 year. Since the number of agents is 100 , each agent has on average 1 opportunity for migration or visit per year.

Figure 1 is an example of the distribution of agents, their networks, and the profit of two agents. Duals form links at two sites. Due to the link formation of 


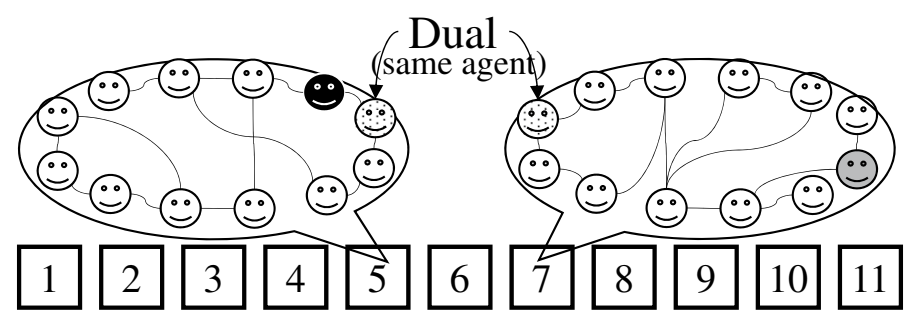

Fig. 1 Distribution of agents at sites 5 and 7 and their network. Dotted agent is dual. The profit of the black agent is $2+5 f+5 f^{2}+3 f^{3}$. The profit of the gray agent is $2+2 f+3 f^{2}+2 f^{3}$

duals, other agents may also profit from indirect links from other sites, even if the agents are singulars.

We define singulars or duals as the role (Simulation 1) or strategy (Simulation 2) of agents. In Simulation 1, whether they are singulars or duals is decided at the initial time and does not change over the simulation. The number of singulars or duals is fixed for the course of the simulation. As independent parameters, we changed the number of duals, or $d$, and the other parameters $(f, p, q)$. The number of duals $d$ is prepared from 0 to 100 , with 10 digits, because the number of agents is 10 for each value of resources $w(6-15)$. For each $w$, we prepare the same number of duals, from 0 to 10; singulars and duals are the same with respect to $w$. During the simulation, duals may select the same site for residence and visits. In that case, they do not behave as duals. We count the number of duals who reside and visit two different sites as active duals; we call the number of active duals $d_{a}$.

In Simulation 2, all agents are at first singulars. The agent, who is already selected for site change, also changes her strategy from singular to dual or vice versa by the probability 0.1 when she changes her site. If the agent has increased her profits after the procedure of the strategy and site change, the new strategy and new site are maintained; if profits have not increased, the agent reverts to her former strategy and site. Even if her strategy is dual, when she selects the same site for residence and visits, her strategy becomes singular in the next time step. We count the number of duals as $d$.

Figure 2 presents the flow chart of the two simulations. The two simulations are almost identical, except for the step of strategy change embedded in Simulation 2.

\section{Results: Simulation 1}

First, we run one-shot simulations for 10,000 turns. We set the values of ( $f, p$, $q)=(0.5,0.3,0.5)$ and change the number of duals, or $d$. In Fig. 3, the number of active duals $\left(d_{a}\right)$ change along turns; dots, dashes, solid and bold curves represent the cases of $d=0,10,30$, and 100, respectively. The figures suggest 2000 turns are suggested for the number of duals to approach equilibrium at all parameter sets. Figure 4 presents the variances of the population at sites for 10,000 turns; dots, dashes, solid and bold curves represent the cases of $d=0,10,30$, and 100, respectively. 


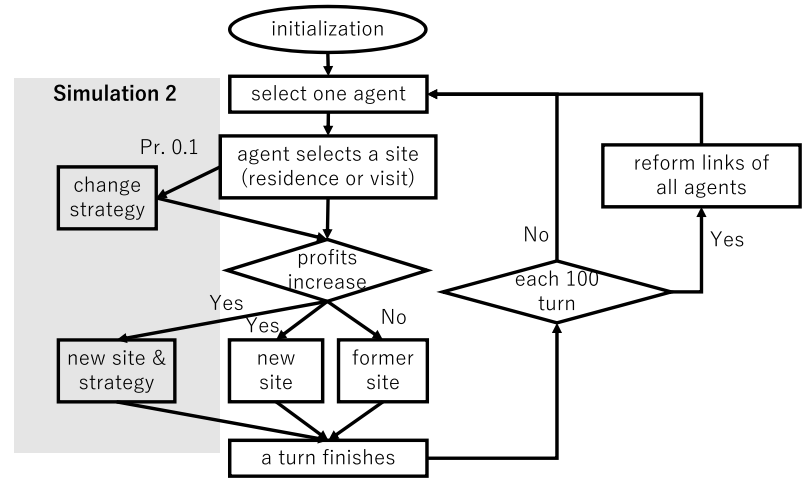

Fig. 2 Flowchart of Simulations 1 and 2

Fig. 3 One-shot simulations for the number of active duals $\left(d_{a}\right)$ in Simulation 1. Dots, dashes, solid, and bold curves represent the case of $d=0,10,30$, and 100 , respectively. $(f, p, q)=(0.5$, $0.3,0.5$ )

Fig. 4 One-shot simulations for the variance of populations in Simulation 1. Dots, dashes, solid, and bold curves represent the case of $d=0,10,30$, and 100 , respectively. $(f, p, q)=(0.5$, $0.3,0.5$ )
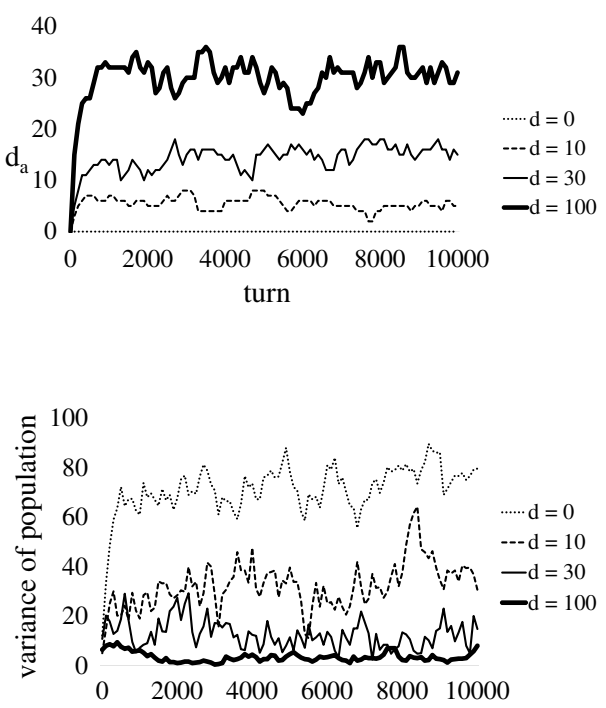

Each one-shot simulation is identical to that represented by Fig. 3. Figure 4 suggests that variances shrink when the number of duals increases. Hereafter, we use the data at turn 2000.

Table 1 shows the numbers, the mean values of resources and profits for singulars, non-active duals and active duals. Each case represents the case of $d=0$, 10, 30, and 100, respectively. Among the agents selected for duals, those with more resources become active and gain higher profits. As the number of duals increases, the mean profits of all agents increase. The table also shows mean values of closeness centrality for the three groups; closeness centrality represents the sum of the length of the shortest paths between the focal agent and all other agents in the network. The closeness centrality of active duals is higher than that of singulars and non-active duals. In addition, closeness centrality becomes larger 
Table 1 Agents for each type: singulars, non-active duals and active duals

\begin{tabular}{lllll}
\hline & $d=0$ & $d=10$ & $d=30$ & $d=100$ \\
\hline Number & & & & \\
$\quad$ Singulars & 100 & 90 & 70 & 0 \\
Non-active duals & 0 & 5 & 18 & 67 \\
Active duals & 0 & 5 & 12 & 33 \\
M.R & & & & \\
Singulars & 10.5 & 10.5 & 10.5 & N.A. \\
Non-active duals & N.A. & 9.6 & 8.9 & 9.3 \\
Active duals & N.A. & 11.4 & 12.8 & 12.9 \\
M.P & & & & \\
Singulars & 7.99 & 10.3 & 14.7 & N.A. \\
Non-active duals & N.A. & 8.8 & 10.9 & 17.1 \\
Active duals & N.A. & 17.7 & 21.9 & 25.2 \\
M.C.C & & & & \\
Singulars & 0.07 & 0.094 & 0.166 & N.A. \\
Non-active duals & N.A. & 0.086 & 0.143 & 0.228 \\
Active duals & N.A. & 0.156 & 0.226 & 0.279 \\
\hline M. & & & & \\
\hline
\end{tabular}

M.R, M.P, and M.C.C, respectively, represent mean resources, mean profits and mean closeness centrality (a)

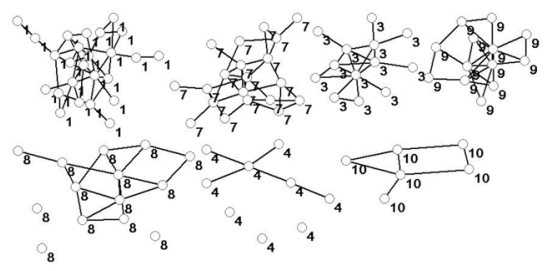

(c)

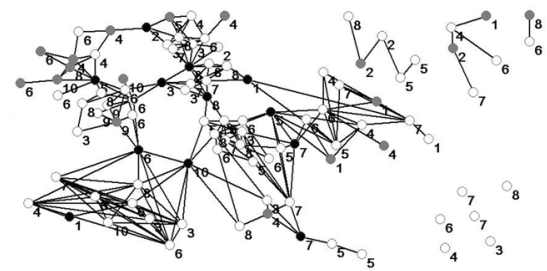

(b)

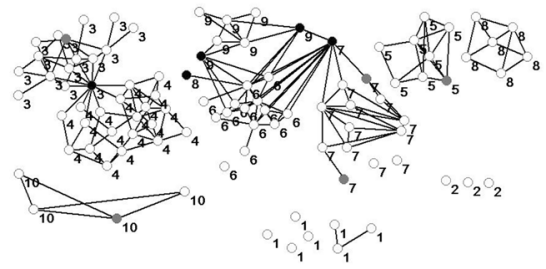

(d)

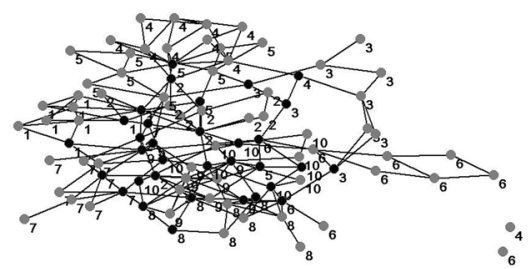

Fig. 5 Social network among agents at turn 2000 in Simulation 1. The blank, gray, and black circles represent singulars, non-active duals, and active duals, respectively. The number represents the residence site of each agent. a $d=0, \mathbf{b} d=10, \mathbf{c} d=30$, and $\mathbf{d} d=100 .(f, p, q)=(0.5,0.3,0.5)$ 
for all the groups as the number of duals increases. Figure 5 presents the social network of agents for each case of (a) $d=0$, (b) $d=10$, (c) $d=30$, and (d) $d=100$. Blank, gray, and black circles, respectively, represent singulars, non-active duals, and active duals. The number at each agent represents the site where each agent resides.

To evaluate inequality between sites, we calculate the mean profits of agents at each residence site. We select the maximum and minimum values of mean profits among the 10 sites, excluding the sites with no agents. We then calculate the inequalities of sites as the difference and the quotient between the maximum and minimum values of the mean profits. We call the two values BSID (between sites inequality difference) and BSIQ (between sites inequality quotient); each value represents the absolute and relative inequality among sites. Table 2 presents the two values in each case of $d=0,10,30$, and 100 , respectively.

We set the value of $f=0.3,0.5$, or 0.7 , and randomly changed the value of $p$ and $q$ with the range of $0.01<p<0.5$ and $0.01<q<1.0$; we check that almost no duals become active when $p>0.5$ or $q>1.0$. We also change the value of $d$ to $0,10, \ldots 100$. For each value of $f$, we run 300 simulations and 900 in total. We check (1) the mean profits and (2) the maximum profits of all agents. We also check (3) BSID and (4) BSIQ. Table 3 presents the results of multiple regression analysis. As the values of $d$ increase, both the mean and maximum profits of agents become large; thus, all agents gain higher profits. Furthermore, as the values of $d$ increases, both BSID and BSIQ decrease; inequality among sites decreases as the number of duals increase. Table 3 also indicates that smaller values of $p$ and $q$ increase the profits of all agents but do not necessarily decrease inequality among sites.

Table 2 Inequality of agents between sites

\begin{tabular}{|c|c|c|c|c|c|c|c|c|}
\hline \multirow[b]{2}{*}{ Site } & \multicolumn{2}{|l|}{$d=0$} & \multicolumn{2}{|l|}{$d=10$} & \multicolumn{2}{|l|}{$d=30$} & \multicolumn{2}{|l|}{$d=100$} \\
\hline & $N$ & M.P & $N$ & M.P & $N$ & M.P & $N$ & M.P \\
\hline 1 & 25 & $\underline{10.8}$ & 7 & 1.7 & 9 & 4.1 & 9 & 20.7 \\
\hline 2 & 0 & - & 3 & 1 & 9 & 10.8 & 11 & 24.6 \\
\hline 3 & 13 & 7.6 & 16 & 13.6 & 0 & - & 9 & 15.6 \\
\hline 4 & 9 & $\underline{3.1}$ & 20 & 14.2 & 20 & 19.8 & 11 & 15.3 \\
\hline 5 & 0 & - & 8 & 6.5 & 16 & 15 & 12 & 20.2 \\
\hline 6 & 0 & - & 14 & 13.3 & 7 & $\underline{22.1}$ & 10 & 14.1 \\
\hline 7 & 20 & 9.3 & 14 & 11.3 & 13 & 16.3 & 10 & 19.4 \\
\hline 8 & 14 & 5.7 & 7 & 5.9 & 9 & 18.8 & 9 & 20.6 \\
\hline 9 & 13 & 8.4 & 7 & 12.2 & 6 & $\underline{3.2}$ & 9 & $\underline{25}$ \\
\hline 10 & 6 & 4.6 & 4 & 3.8 & 11 & 15 & 10 & 22.5 \\
\hline BSID & $10.8-3.1=7.7$ & & $14.2-1.0=13.2$ & & $22.1-3.2=18.9$ & & $25.0-14.1=10.9$ & \\
\hline BSIQ & $10.8 / 3.1=3.5$ & & $14.2 / 1.0=14.2$ & & $22.1 / 3.2=7.0$ & & $25.0 / 14.1=1.8$ & \\
\hline
\end{tabular}

$N$ represents the number of agents at each site. M.P. represents the mean profits of agents at each site. The maximum and minimum values among the 10 sites are highlighted by underline for each case 
Table 3 Results of multiple regression analysis

\begin{tabular}{cccrr}
\hline & Mean profits & Maximum profits & BSID & BSIQ \\
\hline$f=0.3$ & & & & \\
$d$ & 1.36 & 1.97 & -0.52 & -1.34 \\
$p$ & -5 & -7.83 & -2.39 & -0.24 \\
$q$ & -0.51 & -0.77 & 0.16 & 0.001 \\
$R^{2}$ & 0.89 & 0.83 & 0.43 & 0.17 \\
$f=0.5$ & & & & \\
$d$ & 2.95 & 3.57 & -1.09 & -1.93 \\
$p$ & -9.82 & -12 & -2.61 & 0.18 \\
$q$ & -1.02 & -1.27 & 0.11 & -0.28 \\
$R^{2}$ & 0.9 & 0.86 & 0.25 & 0.18 \\
$f=0.7$ & & & & \\
$d$ & 6.35 & 6.94 & -1.84 & -3.13 \\
$p$ & -14.3 & -13.6 & -0.82 & -0.46 \\
$q$ & -2.37 & -2.12 & 0.67 & -0.13 \\
$R^{2}$ & 0.89 & 0.8 & 0.06 & 0.14 \\
\hline
\end{tabular}

Dependent values are mean profits, maximum profits, BSID, and BSIQ. Independent parameters are $d$ : the number of duals, $p$ : the cost of population density, and $q$ : the costs of travel. Each value represents the beta coefficient of the independent parameter. Moreover, the table shows adjusted $R^{2}$ for each multiple regression analysis

\section{Results: Simulation 2}

We run one-shot simulations for 10,000 turns. The values of $(f, p, q)$ are $(0.5,0.3$, $0.5)$, the same as Simulation 1. We add $(0.5,0.1,0.3),(0.7,0.3,0.5)$, and $(0.7,0.1$, $0.3)$ for the values of $(f, p, q)$. The initial number of duals, or $d$, is 0 . In Fig. 6, the number of duals $d$ changes along turns; dots, dashes, solid and bold curves represent the cases of $(f, p, q)=(0.5,0.3,0.5),(f, p, q)=(0.5,0.1,0.3),(f, p, q)=(0.7,0.3,0.5)$, and $(f, p, q)=(0.7,0.1,0.3)$, respectively. Figure 6 suggests that 2000 turns are sufficient for the number of duals to approach equilibrium at all parameter sets. Figures 7 presents variances of the population at sites for 10,000 turns; dots, dashes, solid and bold curves represent the cases of $(f, p, q)=(0.5,0.3,0.5),(f, p, q)=(0.5,0.1,0.3)$, $(f, p, q)=(0.7,0.3,0.5)$, and $(f, p, q)=(0.7,0.1,0.3)$, respectively. Each one-shot simulation is identical to that in Fig. 6 . Since the variances change intensively along

Fig. 6 One-shot simulations for the number of duals $(d)$ in Simulation 2. Dots, dashes, solid, and bold curves represent the case of $(f, p, q)=(0.5,0.3$, $0.5),(f, p, q)=(0.5,0.1,0.3),(f$, $p, q)=(0.7,0.3,0.5)$, and $(f, p$, $q)=(0.7,0.1,0.3)$, respectively

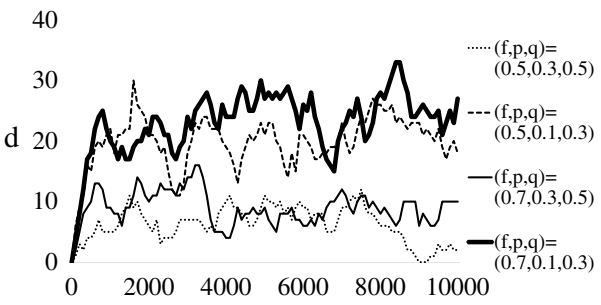


Fig. 7 One-shot simulations for the variance of populations in Simulation 2. Dots, dashes, solid, and bold curves represent the case of $(f, p, q)=(0.5,0.3$, $0.5),(f, p, q)=(0.5,0.1,0.3),(f$, $p, q)=(0.7,0.3,0.5)$, and $(f, p$, $q)=(0.7,0.1,0.3)$

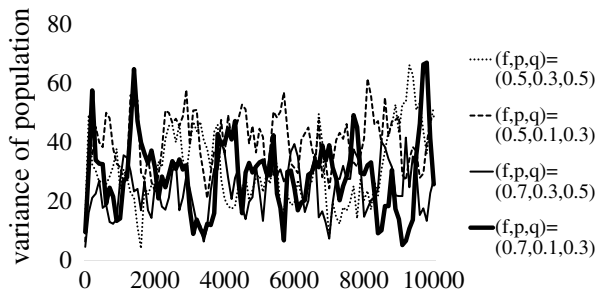

turns in any case, we cannot deduce some findings from the figure. Hereafter, we use the data at turn 2000.

Table 4 shows the numbers, the mean values of resources and profits for singulars and duals. Each case represents the case of $(f, p, q)=(0.5,0.3,0.5),(f, p, q)=(0.5$, $0.1,0.3),(f, p, q)=(0.7,0.3,0.5)$, and $(f, p, q)=(0.7,0.1,0.3)$, respectively. In each case of $f$, as the costs of density and travel ( $p$ and $q$ ) decrease, more agents become duals and mean profits of all agents increase. The table also shows mean values of closeness centrality for the two groups. Closeness centrality is higher in duals than in singulars. Closeness centrality becomes larger even for singulars as the costs of density and travel decrease, or the number of duals increase. Figure 8 presents the social network of agents for the case of (a) $(f, p, q)=(0.5,0.3,0.5)$, (b) $(f, p, q)=(0.5,0.1$, $0.3),(\mathrm{c})(f, p, q)=(0.7,0.3,0.5)$, and (d) $(f, p, q)=(0.7,0.1,0.3)$, respectively. Blank and black circles, respectively, represent singulars and duals. The number at each agent represents the site where each agent resides.

We set the value of $f=0.3,0.5$, or 0.7 and randomly changed the value of $p$ and $q$ within the range of $0.01<p<0.5$ and $0.01<q<1.0$. To test the effects of the independent parameters on the evolution of duals, we checked the number of duals, the

Table 4 Agents for each type: singulars and duals

\begin{tabular}{lllll}
\hline & $(f, p, q)=$ & $(f, p, q)=$ & $(f, p, q)=$ & $(f, p, q)=$ \\
& $(0.5,0.3,0.5)$ & $(0.5,0.1,0.3)$ & $(0.7,0.3,0.5)$ & $(0.7,0.1,0.3)$ \\
\hline $\begin{array}{llll}\text { Number } \\
\text { Singulars }\end{array}$ & 94 & & & \\
Duals & 6 & 79 & 90 & 79 \\
M.R & & 21 & 10 & 21 \\
Singulars & 10.4 & 10.3 & 10.2 & 10.2 \\
Duals & 12.8 & 11.4 & 12.8 & \\
M.P & & & & 49.3 \\
Singulars & 10 & 26.9 & 22.9 & 56.2 \\
Duals & 16.5 & 34.5 & 34.1 & \\
M.C.C & & & 0.172 & 0.311 \\
Singulars & 0.099 & 0.296 & 0.225 & 0.35 \\
Duals & 0.147 & 0.343 & & \\
\hline
\end{tabular}

M.R, M.P, and M.C.C, respectively, represent mean resources, mean profits and mean closeness centrality 
(a)

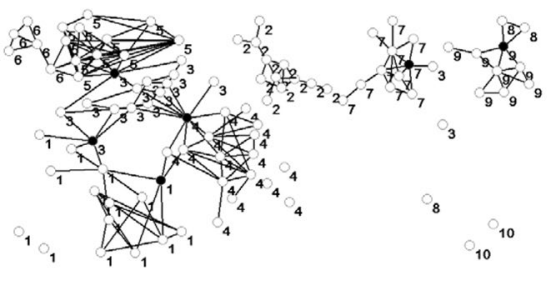

(c)

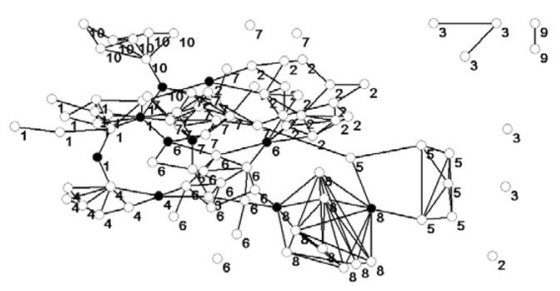

(b)

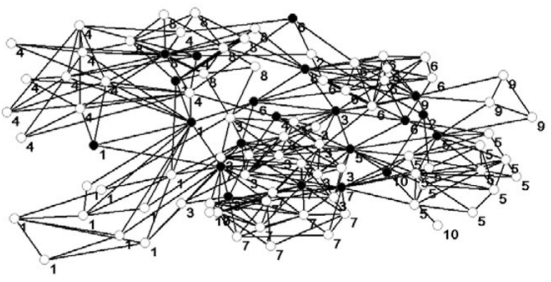

(d)

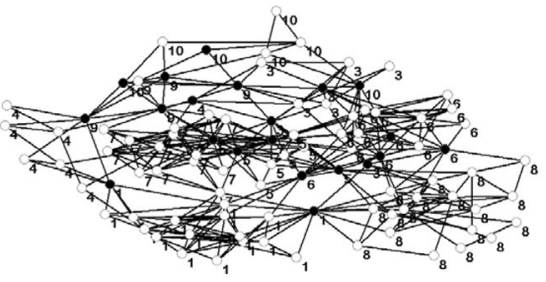

Fig. 8 Social network among agents at turn 2, turn in Simulation 2. Blank and black circles represent singulars and active duals, respectively. The number represents the residence site of each agent. a $(f, p$, $q)=(0.5,0.3,0.5), \mathbf{b}(f, p, q)=(0.5,0.1,0.3), \mathbf{c}(f, p, q)=(0.7,0.3,0.5)$, and $\mathbf{d}(f, p, q)=(0.7,0.1,0.3)$

mean profits of singulars, and the mean profits of duals. For each value of $f$, we run 300 simulations and 900 in total. In some simulations, no agents become duals. We exclude the case when there are no duals and use the data of 206 for $f=0.3,258$ for $f=0.5$ and 286 for $f=0.7$. Figure 9 presents the results of pass analysis: (a) $f=0.3$, (b) $f=0.5$, and (c) $f=0.7$. In each case, as the costs of density and travel decrease, the number of duals increases. Not only duals but also singulars gain higher profits as the costs of density and travel decrease, and as the number of duals increases.

To expect the mean resources of duals, we include all the other parameters as independent parameters and select the best-fit generalized linear model based on

(a)

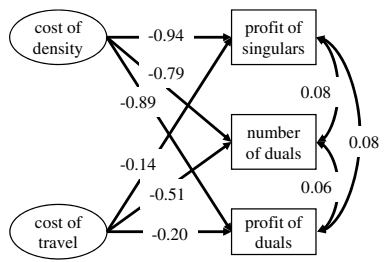

(b)

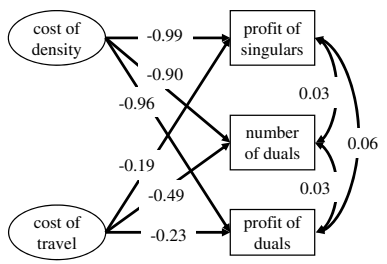

(c)

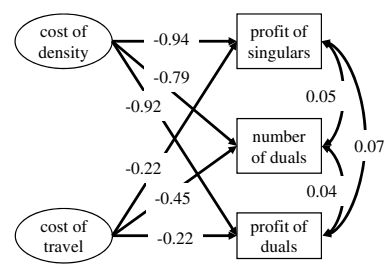

Fig. 9 The results of path diagram, within the range of $0.01<p<0.5$ and $0.01<q<1.0$. a $f=0.3$, b $f=0.5$, and $\mathbf{c} f=0.7$. For each case, we run 300 simulations. We delete the data with no singulars or no duals. The number of simulation data is a 206, b 258, and c 286, respectively. All the values are standardized 
AIC. The values $r_{D}, g_{D}, g_{S}$, and $d^{*}$, respectively represent the mean resources of duals, mean profits of duals, mean profits of singulars, and number of duals. If duals evolve from any agents independent of their resources, $r_{D}$ should become 10.5 (mean value of 6-15). As we do in Fig. 9, we delete the case with no duals and use the data of 206 for $f=0.3,258$ for $f=0.5$, and 286 for $f=0.7$. In each case of (a) $f=0.3$, (b) $f=0.5$, and (c) $f=0.7$, the best-fit model was (a) $r_{D}=9.36+4.0 p+0.32$ $g_{D}-0.24 g_{S}-0.067 d^{*}(\mathrm{AIC}=65.0),(\mathrm{b}) r_{D}=9.39+6.1 p+0.18 g_{D}-0.13 g_{S}-0.054$ $d^{*}(\mathrm{AIC}=87.3)$, and (c) $r_{D}=9.93+5.1 p+1.1 q+0.10 g_{D}-0.10 g_{S}(\mathrm{AIC}=-127.7)$. To check the effects of the two independent values of $p$ and $q$, we substitute the mean values of $g_{D}, g_{S}$, and $d^{*}$ : they are (a) 17.7, 12.5, and 10.7, (b) 24.5, 18.4, and 10.9 , (c) $37.0,30.9$, and 12.5, respectively. We gain equations (a) $r_{D}=11.31+4.0 p$, (b) $r_{D}=10.82+6.1 p$, and (c) $r_{D}=10.54+5.1 p+1.1 q$. Although the values of $r_{D}$ are always larger than 10.5, agents with small resources may become duals when the costs of density and travels are small.

\section{Discussion}

In the age of globalization, individuals with rich resources are more likely to have opportunities to move across boundaries of countries, reside in profitable sites, and gain more profits. The areas attract residents and capital. As population density increases, the cost of living increases. Poor residents cannot afford to live there because of the high cost and are displaced to areas of low population density. Inequalities among sites may expand, and only super-rich individuals may gain exclusive benefits. The lifestyle of bohemians and tourists, in this paper modeled as dual, may mitigate inequalities among sites and increase the profits of all parties. By contrast, the lifestyle may expand inequality.

In Simulation 1, we changed the number of duals and test their effects on the social network among agents. Duals should select sites of many agents to increase their profits. As the number of duals increases, duals do not visit the sites of many agents because other duals already have links at the sites. By contrast, duals visit the sites of few agents to further extend their social network. The extended social network includes more agents. Thus, duals increase their profit and the profits of all agents. Consequentially, the agents at any site gain higher profits and the inequalities among sites decrease as the number of duals increases. The variance of population also shrinks as the number of duals increases, because agents can gain profits at any site. Therefore, the hypothesis that duals mitigate inequality and increase all profits is supported. When the costs of population density and travel are smaller, agents also gain higher profits, but inequality among sites does not necessarily decrease. Although duals gain relatively higher profits than singulars, they pay the additive costs of density and travel. Hence, duals might be regarded as altruistic toward all other agents.

In Simulation 2, agents become singulars or duals as their strategy. Agents with more resources become duals and gain more profits than singulars. Inequality among agents may widen because of the possibility of the dual strategy. However, as the number of duals increases, not only duals but also singulars gain higher profits. 
Furthermore, agents with smaller resources may become duals as costs of density and travel decrease. The costs of population density and travel are exogenous independent parameters in the present model. If agents connected to an extended social network and higher profits could decrease the costs through innovation, the strategy of duals may be more open to agents with few resources.

If individuals are satisfied with social relations only within their residence areas, they rarely communicate with individuals from distant areas; outsiders are excluded from communication. Individuals are often linked by homophile, based on, for example, age, culture, education, income, or locations. The benefits from dense and closed networks within a group that excludes outsiders are, for example, improved human capital [18], shared governance of common property resources [46], trust between members [23], and a healthy life [33]. Dense and closed networks between individuals of the same group, however, may also have negative consequences such as resistance to new ideas, xenophobia, and cultural impoverishment [48]. In this paper, homophile networks are formed when the number of duals is small. Agents with many resources can form networks with one another in a site of many fellows while excluding agents of few resources. Agents with few resources cannot reside in sites with many agents, because of high cost; they reside in sites of few fellows and gains little profits. Inequality among sites should become large, which are also the negative consequences for society.

Some individuals who specialize in bridging groups have played key roles in today's society where individuals are freed from locations. The specialists should be called coordinators, brokers, gatekeepers, representatives, or liaisons, depending on their social positions [25]. Their advantage could be because of globalization; they are not required to embed in a restricted area. Weak ties [26, 27] are expected to bridge groups or areas. Individuals with weak ties may gain exclusive benefits, which should surpass the cost of maintaining the position of structural holes [11, 12]. The lifestyle of bohemians and tourists, modeled in this paper as dual, may connect individuals of different sites in a more extant network. Networks across areas provide several benefits. Social networks across groups often contribute to community development, and communities remain available for locally restricted residents. Historically, communities have developed by welcoming outsiders to promote democratic institutions and support new economic activities. Areas with a high tolerance of outsiders and minorities flourish because of the diversity of individuals and creative businesses that emerge from collaborations between diverse sets of individuals [21, 50]. Although Boorstin [10] described tourists as individuals seeking superficial pseudoevents, many tourists sincerely search for authentic experiences and form links with other tourists and residents while traveling [16, 40]. Bohemians and tourists could form links in their targeted areas while maintaining their original links in the areas of their residences. The areas may develop because of the bohemians and tourists' lifestyles. Residents increase their quality of life and enhance the creativity of the area by promoting high tolerance and high productivity. Nowadays, an increasing number of bohemians and tourists visit destinations worldwide. Understanding their network formation is difficult. Their effects on network formation have rarely been studied [17]. Complexity science can explain how networks of destinations, organizations, or influxes of tourists occur [3]. In that sense, the 
present $\mathrm{ABS}$ and further research could explore how tourism contributes to network formation across distant areas.

Globalization often causes negative effects on local communities, such as destroying cooperation or local cultures, because individuals from distant areas are strangers and do not follow the rules of local communities as much as residents do. However, studies have demonstrated that interactions of strangers may result in positive outcomes. The evolution of cooperation is possible through interaction among distant agents. Anonymous individuals aggregated from distant areas could share their resources [28], and this case is often found on occasions of great disaster [58]. Due to interaction across distant sites, individuals can retain multiple cultural traits or accumulate multiple knowledge locally embedded in each site [14, 29]. This paper demonstrates that social networks across distant sites not only increase the profits of all members but also shrink inequality among sites. Further research may find other positive effects of globalization, which promote individuals of distant sites to interact more.

Our ABS has several limitations. First, the model assumes that the value of $f$ is fixed for all agents; thus, we could have relaxed this assumption and assigned different values to agents. Depending on their age, job, sex, or social capital, individuals can have a range of social relations. Depending on the values of $f$, agents-singulars or duals-may select their strategies. Second, in the model, the resources of agents are fixed during the simulation. We may assume that agents with more profits gain high resources at the next turn; then, inequalities among agents with respect to resources and profits may change as time pass. Last, our model did not assume negative or positive effects of closed or open social networks. Further research may add factors by considering the diversity of sites of an individual connected by her network. Nevertheless, the present model demonstrated the results of a minimalist model and applied the KISS principle, "Keep It Simple, Stupid.” Further research could gain deeper results by incorporating additive factors and data with clear assumptions.

Acknowledgements This research was supported by the MEXT GRANT 16H03698, 16K04028, and 16K07510. I thank M. Asaoka, Y. Ihara, G. Kamada, Y. Kanazawa, W. Nakahashi, Y. Nakai, and K. Tominaga for valuable comments and discussions. I also thank four anonymous reviewers who give critical and constructive comments on the manuscript. The author would like to thank Enago (www.enago.jp) for the English language review.

Open Access This article is licensed under a Creative Commons Attribution 4.0 International License, which permits use, sharing, adaptation, distribution and reproduction in any medium or format, as long as you give appropriate credit to the original author(s) and the source, provide a link to the Creative Commons licence, and indicate if changes were made. The images or other third party material in this article are included in the article's Creative Commons licence, unless indicated otherwise in a credit line to the material. If material is not included in the article's Creative Commons licence and your intended use is not permitted by statutory regulation or exceeds the permitted use, you will need to obtain permission directly from the copyright holder. To view a copy of this licence, visit http://creativecommons.org/ licenses/by/4.0/. 


\section{References}

1. Alizadeh, M., Cioffi-Revilla, C., \& Crooks, A. (2016). Generating and analyzing spatial social networks. Computational and Mathematical Organization Theory, 23(3), 362-390.

2. Allen, B., Lippner, G., Chen, Y., Fotouhi, B., Momeni, N., Yau, S., \& Nowak, M. A. (2017). Evolutionary dynamics on any population structure. Nature, 544(7649), 227-232.

3. Baggio, R. (2008). Symptoms of complexity in a tourism system. Tourism Analysis, 13(1), 1-20.

4. Barabasi, A., \& Albert, R. (1999). Emergence of scaling in random networks. Science, 286(5439), 509-512.

5. Bauman, Z. (2001). Community: seeking safety in an insecure world. Polity.

6. Benard, S., \& Willer, R. (2007). A wealth and status-based model of a residential segregation. Journal of Mathematical Sociology, 31(2), 149-174.

7. Bergé, L. R. (2017). Network proximity in the geography of research collaboration. Papers in Regional Science, 96(4), 785-815.

8. Bettencourt, L. M. A. (2013). The origins of scaling in cities. Science, 340(6139), 1438-1440.

9. Bianchi, F., \& Squazzoni, F. (2015). Agent-based models in sociology. WIREs Computational Statistics, 7(4), 284-306.

10. Boorstin, D. J. (1962). The image: a guide to pseudo-events in America. NewYork: Atheneum.

11. Burt, R. (1992). Structural holes: the social structure of competition. Cambridge: Harvard University Press.

12. Burt, R. (2005). Brokerage and closure: an introduction to social capital. Oxford: Oxford University Press.

13. Buskens, V., \& Rijt, A. (2008). Dynamics of networks if everyone strives for structural holes. American Journal of Sociology, 114(2), 371-407.

14. Centola, D., Gonzalez-Avella, J. C., Eguiluz, V. M., \& Miguel, M. S. (2007). Homophily, cultural drift, and the co-evolution of cultural groups. Journal of Conflict Resolution, 51(6), 905-929.

15. Cho, J. N., Mansury, Y. S., \& Ye, X. (2016). Churning, power laws, and inequality in a spatial agentbased model of social networks. The Annals of Regional Science, 57(2-3), 275-307.

16. Cohen, E. (1988). Authenticity and commoditization in tourism. Annals of Tourism Research, 15(3), 371-386.

17. Cohen, E., \& Cohen, S. A. (2012). Current sociological theories and issues in tourism. Annals of Tourism Research, 39(4), 2177-2202.

18. Coleman, J. S. (1988). Social capital in the creation of human capital. American Journal of Sociology, 94, S95-S120.

19. Dunbar, R. (1998). Grooming, gossip, and the evolution of language. Cambridge: Harvard University Press.

20. Ezcurra, R., \& Rodriguez-Poze, A. (2013). Does economic globalization affect regional inequality? a cross-country analysis. World Development, 52, 92-103.

21. Florida, R. (2012). The rise of the creative class revisited. New York: Basic Books.

22. Frenzel, F., Koens, K., \& Steinbrink, M. (Eds.). (2012). Slum tourism: poverty, power and ethics. London: Routledge.

23. Fukuyama, F. (1995). Trust: the social virtues and the creation of prosperity. New York: Free Press Paperbacks.

24. Glückler, J. (2007). Economic geography and the evolution of networks. Journal of Economic Geography, 7(5), 619-634.

25. Gould, R. V., \& Fernandez, R. M. (1989). Structure of mediation: formal approach to brokerage in transaction networks. Sociological Methodology, 19, 89-126.

26. Granovetter, M. S. (1973). The strength of weak tie. American Journal of Sociology, 78(6), 1360-1380.

27. Granovetter, M. S. (1974). Getting a job: a study of contacts and careers. Cambridge: Harvard University Press.

28. Horiuchi, S. (2015). Emergence and collapse of the norm of resource sharing around locally abundant resources. Journal of Artificial Societies and Social Simulation, 18(4), 7.

29. Horiuchi, S., \& Takakura, J. (2019). Modeling learning strategies and the expansion of the social network in the beginning of upper paleolithic Europe: analysis by agent-based simulation. In Y. Nishiaki \& O. Joris (Eds.), Learning behaviors among neanderthals and paleolithic modern humans: an introduction (pp. 179-191). Tokyo: Springer. 
30. Jacobs, J. (1961). The death and life of great American cities. London: Random House Publishing.

31. Jackson, M. O., \& Wolinsky, A. (1996). A strategic model of social and economic networks. Journal of Economic Theory, 71(1), 44-74.

32. Jackson, J., Forest, B., \& Sengupta, R. (2008). Agent-based simulation of urban residential dynamics and land rent change in a gentrifying area of Boston. Transactions in GIS, 12(4), 475-491.

33. Kawachi, I., Takao, S., \& Subramanian, S. V. (2003). Global perspectives on social capital and health. New York: Springer.

34. Kim, K., \& Altman, J. (2017). Effect of homophily on network formation. Communications in Nonlinear Science and Numerical Simulation, 44, 482-494.

35. Kossinets, G., \& Watts, D. J. (2006). Empirical analysis of an evolving social network. Science, 311(5757), 88-90.

36. Krugman, P. (1991). Increasing returns and economic geography. Journal of Political Economy, 99(3), 483-499.

37. Li, R., Dong, L., Zhang, J., Wang, X., Wang, W., Di, Z., \& Stanley, H. E. (2017). Simple spatial scaling rules behind complex cities. Nature Communications, 8(1), 1-7.

38. Liben-Nowell, D., Novak, J., Kumar, R., Raghavan, P., \& Tomkins, A. (2005). Geographic routing in social networks. Proceedings of the National Academy of Sciences of the United States of America, 102(33), 11623-11628.

39. Lucas, R. E., Jr. (2004). Life earnings and rural-urban migration. Journal of Political Economy, 112, S29-S59.

40. MacCannell, D. (1976). The tourist: a new theory of the leisure class. California: University of California Press.

41. Mansury, Y., \& Shin, J. K. (2015). Size, connectivity, and tipping in spatial networks: Theory and empirics. Computers, Environment and Urban Systems, 54, 428-437.

42. Masuda, H. (2014). Chifo Shometsu (Regioal Areas Disspeaing). Tokyo: Chuo Koron Sha.

43. McIntyre, N., Williams, D., \& McHugh, K. (Eds.). (2006). Multiple dwelling and tourism: negotiating place, home and identity. Oxford: Cabi.

44. McPherson, M., Smith-Lovin, L., \& Cook, J. M. (2001). Birds of a feather: homophily in social networks. Annual Review of Sociology, 27(1), 415-444.

45. Nowak, M. A., \& May, R. M. (1992). Evolutionary games and spatial chaos. Nature, 359(6398), $826-829$.

46. Ostrom, E. (1990). Governing the commons: the evolution of institutions for collective action. Cambridge: Cambridge University Press.

47. Pan, W., Ghoshal, G., Krumme, C., Cebrian, M., \& Pentland, A. (2013). Urban characteristics attributable to density-driven tie formation. Nature Communications, 4(1), 1961.

48. Portes, A. (1998). Social capital: its origin and applications in modern sociology. Annual Review of Sociology, 24(1), 1-24.

49. Prell, C., \& Lo, Y. (2016). Network formation and knowledge gains. The Journal of Mathematical Sociology, 40(1), 21-52.

50. Putnam, R. D. (2000). Bowling alone: the collapse and revival of American community. New York: Simon \& Schuster.

51. Sahasranaman, A., \& Jensen, H. J. (2016). Dynamics of transformation from segregation to mixed wealth cities. PlosOne, 11(11), e0166960.

52. Sahasranaman, A., \& Jensen, H. J. (2017). Cooperative dynamics of neighborhood economic status in cities. PlosOne, 12(8), e0183468.

53. Sassen, S. (2001). The global city: New York, London, Tokyo. Oxford: Princeton University Press.

54. Schläpfer, M., Bettencourt, L. M. A., Grauwin, S., Raschke, M., Claxton, R., Smoreda, Z., et al. (2014). The scaling of human interactions with city size. Journal of Royal Society Interface, 11(98), 20130789.

55. Schelling, T. C. (1971). Dynamic models of segregation. Journal of Mathematical Sociology, 1(2), 143-186.

56. Skyrms, B., \& Pemantle, R. (2000). A dynamic model of social network formation. Proceedings of the National Academy of Sciences of the United States of America, 97(16), 9340-9346.

57. Smith, N. (1996). The new urban frontier: Gentrification and the revanchist city. London: Routledge.

58. Solnit, R. (2009). A paradise build in hell: the extraordinary communities that arise in disaster. New York: Viking. 
59. Todaro, M. P. (1969). A model of labor migration and urban unemployment in less developed countries. The American Economic Review, 59(1), 138-148.

60. Topirceanu, A., Udrescu, M., \& Marculescu, R. (2018). Weighted betweenness preferential attachment: a new mechanism explaining social network formation and evolution. Scientific Reports, 8(1), $1-14$.

61. Torrens, P. M., \& Nara, A. (2007). Modeling gentrification dynamics: a hybrid approach. Computers, Environment and Urban Systems, 31(3), 337-361.

62. Watts, D. J., \& Strogatz, S. H. (1998). Collective dynamics of 'small-world' networks. Nature, 393(6684), 440-442.

Publisher's Note Springer Nature remains neutral with regard to jurisdictional claims in published maps and institutional affiliations. 